\title{
Comparison of the possible side effects of cyclosporine and tacrolimus on renal function in kidney transplant patients
}

\author{
Mohammed abdul muttalib abdul bari \\ Baghdad collage of medical sciences \\ E-Mail: Mohammed_abdulmutalib@yahoo.com
}

\begin{abstract}
Background: Cyclosporine and Tacrolimus are two main immunosuppressants of kidney transplant patients and are commonly used in hospitals with renal transplantation.

Aim of the study: The goal of this study is to demonstrate potential harmful effects, not only from kidney failure as a disease, but also the use of immunosuppressants before and after renal transplants.

Materials and Methods: An analysis of tacrolimus or cyclosporine immunosuppressive regimes has been conducted in Baghdad hospital using data from patients with kidney transplants. The study excluded patients who received other types of immune suppressant drugs, and those who switched from cyclosporine to tacrolimus.

Conclusion: Effectiveness and side effects should be tested in kidney transplantation patients with cyclosporin and tacrolimus and other immunosuppressive medications, tacrolimus treated patient has shown an increase in glucose level and decrease in WBC, hemoglobin and calcium level in comparing to cyclosporine treated group.
\end{abstract}

This finding may allow scientists and physicians to make a better choice between immunosuppressive drug s according to the patient's medical condition.

Keywords: Cyclosporine, Tacrolimus, renal failure, renal transplant.

\section{Introduction}

Chronic kidney disease [CKD] is a major threat to public health with a rapid rise in its global prevalence and occurrence, particularly in the developing countries, resulting in end-stage renal disease [ESRD], premature mortality, poor quality of life and large health systems burden. There are currently clear guidelines and an internationally agreed system for CKD staging that facilitates diagnosis and management of the CKD amongst the nations and populations involved in the studies [1,2].

In most instances, a prolonged path of gradual renal function loss is followed by end stage renal disease. The population with earlier stages of CKD is an ideal target for interventions to slow progression to ESRD and for optimizing management of co-morbid conditions, e.g. cardiovascular disease, anemia, etc., in order to improve long-term outcomes [3].

As an end result of the rise in non-communicable diseases, the number of patients with end stage kidney disease [ESKD] is growing worldwide. ESKD is a medical condition for a lifetime which has a significant impact on clinicians, communities and the health sector. Over 2 million people worldwide have been estimated to need life support therapy for ESKD. In reality the number might be 10 folds higher than the estimated figures as the access to renal replacement therapy [RRT] is restricted or not available in over 112 countries and before accessing diagnostic and therapeutic health services, many patients die [4].

The diagnosis of renal failure in addition to physical exams and complete medical history may include: blood and urine analysis, ultrasound and kidney biopsy [5].

End stage renal failure has a number of different causes. Chronic renal disease risk factors include growing of age, type II diabetes mellitus and hypertension. In contrast to glomerulonephritis, polycystic kidney, atherosclerosis and obstructive uropathy, untreated hypertension is the most common cause [6].

Renal transplantation is a preferred cure for a minority of patients with ESRD. Improved early graft survival and long term graft function have increased the cost-effectiveness of kidney transplantation as an alternative to dialysis [7]. 
The defense against harmful foreign molecules is the importance of the immune system. However, this protection may in some cases lead to a serious problem. For example, the introduction of an allograft can bring out a damaging immune response, causing rejection of the transplanted tissue [8].

Two of a major immunosuppresent drugs that is used for kidney transplant patient is Cyclosporine and Tacrolimus . Cyclosporin consisted of eleven amino acids it's a lipophilic cyclic polypeptide used to prevent rejection of the kidney, liver and heart transplant allogeneic. By combination with corticoids and antimetabolite drugs, cyclosporine is most effective in preventing the acute rejection of transplanted organs. Cyclosporine appears to inhibit immune cell induced responses, whereas humeral immunity is much less impaired. Treatment may be linked to several potentially serious side effects: enlarged gum, seizures, peptic ulcers, fever, vomiting, high blood pressure nephrotoxicity, hypercholesterolemia and hepatotoxicity [9].

Tacrolimus, On the other hand, is approved for rejection protection for liver and kidney transplant and is administered with a corticosteroid or anti-metabolites. This medicine was found to be beneficial in compare to cyclosporine because of its potential and decreases in rejection and also because less corticosteroid doses are used with it. , thus decreasing the likelihood of steroid-associated side effects. Tacrolimus performs like cyclosporin in its immunosuppression reaction, except that it bind to a different immunophilin .and also a wide numbers of side effects are recognized vary between nephrotoxicity and anaphylactic reactions [10].

The purpose of the study is to highlight the potential serious effect not only from renal failure but also from the use of immunosuppressive drugs after renal transplantation, and the safety differences between these drugs.

\section{Materials and methods}

A study of tacrolimus or cyclosporine immunosuppressive regimens in the Baghdad clinics has been performed using evidence from patients undergoing kidney transplantation.

Also the study included only incident patients who underwent transplantation in 2015 and were followedup for at least 3 months.

2 Patient was from both gender and age from (40 -65) included in this study Patients who received more types of immune suppressant drugs than that we include in this study was excluded and those who switched from cyclosporine to tacrolimus-based regimens.

SPSS22 analytical method used to estimate the p value. A significance level of 5\% was considered.

\section{Group design}

Renal transplant Patients was divided into 2 main groups:

Group (1): 11 patient received cyclosporine as there immunosuppressant drug in addition to corticosteroid.

Group (2): 11 patient received Tacrolimus as there immunosuppressant drug in addition to corticosteroid.

\section{Laboratory analysis}

All patients had been examined after the kidney transplantation for kidney function tests, glucose level and electrolyte concentration as follows:

1- Creatinine serum concentration.

2- Urea blood concentration.

3- Glucose blood concentration.

4- Sodium serum concentration.

5- Potassium serum concentration.

6- Calcium serum concentration.

7- WBC count.

8- Platelet count.

9- Hemoglobin level. 


\section{Results}
A. Biochemical results

Table (1): the means of biochemical parameters levels in group $(1,2)$ in patient after kidney transplant

\begin{tabular}{|c|c|c|c|}
\hline & Normal values & Group 1 & Group 2 \\
\hline Glucose levels mg/dl & $70-100$ & $137.88 \pm 21.54$ & $147.66 \pm 24.5^{*}$ \\
\hline UREA levels mg/dL & $8-21$ & $79.1 \pm 19.9$ & $85.6 \pm 34.0$ \\
\hline Creatinine levels mg/dL & $0.8-1.3$ & $2.7 \pm 0.95$ & $2.5 \pm 1.2$ \\
\hline WBC levels/ $\mu \mathrm{L}$ & $4000-10,000$ & $12388.8 \pm 4168.16$ & $7066.66 \pm 2003.33 *$ \\
\hline Platelet levels/ $\mu \mathrm{L}$ & $\mathbf{1 5 0 , 0 0 0 - 3 5 0 , 0 0 0}$ & $\mathbf{2 3 4 0 0 0 \pm 8 5 2 8 7 . 7 4}$ & $156000 \pm 121622.36 *$ \\
\hline Hemoglobin levels g/dL & $14-17$ & $\mathbf{8 . 2 5 5 6} \pm 2.4$ & $\mathbf{6 . 9 3 3 3} \pm 1.5$ \\
\hline
\end{tabular}

Group (1): cyclosporine treated group, Group (2): Tacrolimus treated group * represent a significant deference between group (1) and group (2) [p<0.05] SPSS22 analytical method used to estimate the p value.

\section{B. Electrolyte results}

Table (2): the means of electrolyte levels in group $(1,2)$ and $(3)$ in patient after kidney transplant

\begin{tabular}{|c|c|c|c|c|c|c|c|c|c|}
\hline & $\begin{array}{c}\text { Normal } \\
\text { Ca level } \\
\text { mg/dL }\end{array}$ & $\begin{array}{c}\text { Ca1 } \\
\text { Group } \\
1\end{array}$ & $\begin{array}{c}\mathrm{Ca} 2 \\
\text { Group } \\
2\end{array}$ & $\begin{array}{c}\text { Norml } \\
\mathrm{K} \\
\text { level } \\
\text { meq/L }\end{array}$ & $\begin{array}{c}\text { K1 } \\
\text { Group } \\
1\end{array}$ & $\begin{array}{c}\text { K2 } \\
\text { Group } \\
2\end{array}$ & $\begin{array}{c}\text { Normal } \\
\text { sodium } \\
\text { level } \\
\text { meq/L }\end{array}$ & $\begin{array}{c}\text { Na1 } \\
\text { Group } \\
1\end{array}$ & $\begin{array}{c}\mathrm{Na} 2 \\
\text { Group } \\
2\end{array}$ \\
\hline $\begin{array}{c}\text { Mean } \\
\text { Electrolyte }\end{array}$ & $9-10.5$ & $\begin{array}{l}8.6 \pm \\
0.28\end{array}$ & $\begin{array}{c}7.7 \pm \\
0.01 *\end{array}$ & $\begin{array}{c}3.5- \\
5.0\end{array}$ & $\begin{array}{c}4.17 \pm \\
1.1\end{array}$ & $\begin{array}{l}3.8 \pm \\
0.55\end{array}$ & $136-145$ & $\begin{array}{c}139.2 \pm \\
4.34\end{array}$ & $\begin{array}{l}134 \pm \\
0.001\end{array}$ \\
\hline
\end{tabular}




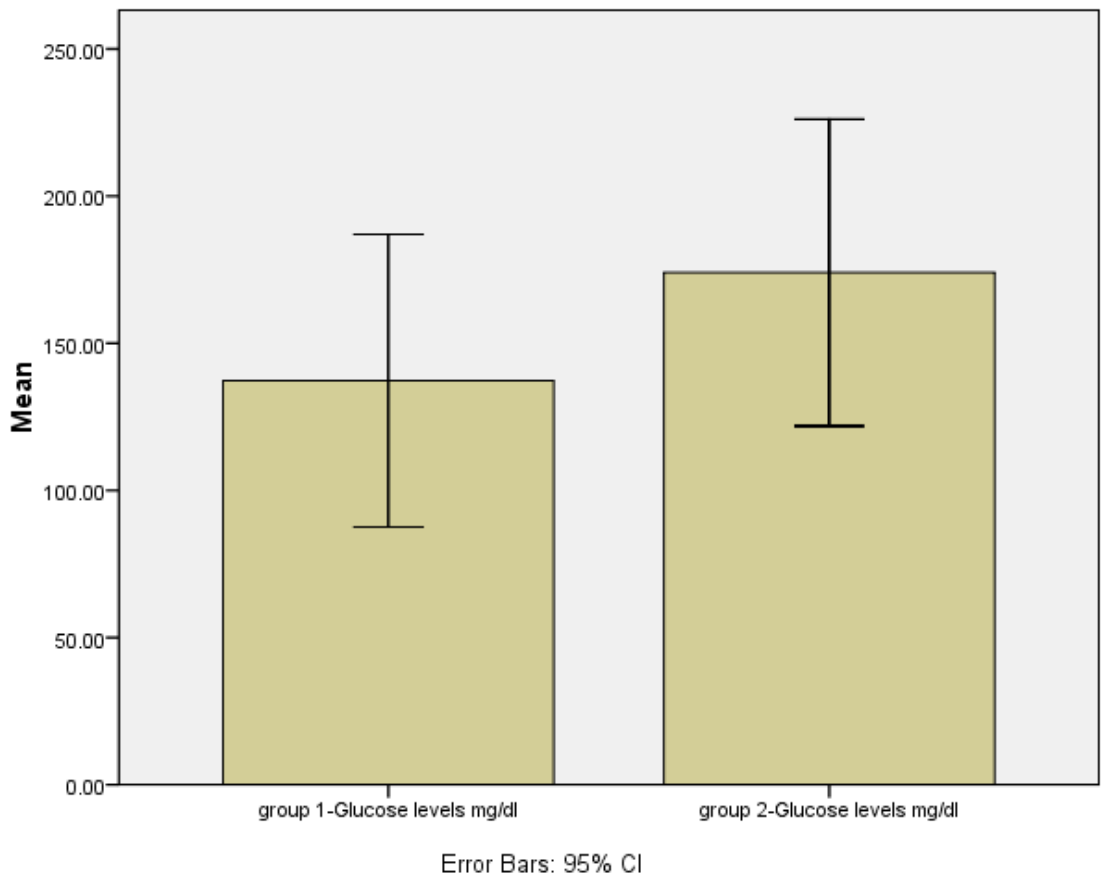

Figure (1): represent the differences between group (1) and (2) in glucose level after 3 months of kidney transplant surgery

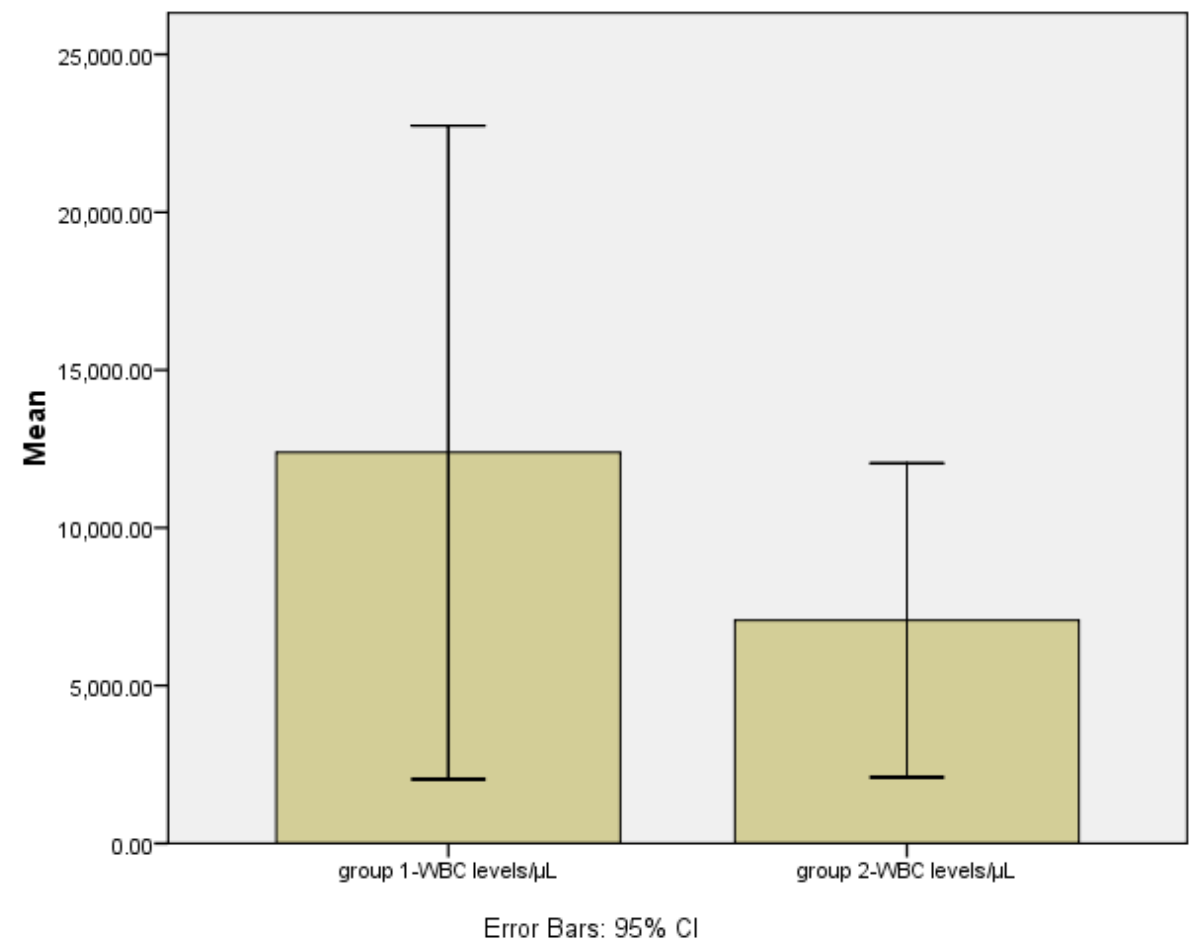

Figure (2): represent the differences between group (1) and (2) in WBC count after 3 months of kidney transplant surgery 


\section{Discussion}

Tacrlimus and cyclosporine are thought to contributed to Hyperglycemia through a variety of mechanism , including the inducement of insulin resistance coupled with direct beta cell effects, The differential impact of individual immunosuppressive agents on incidence of PTDM[ post-transplant diabetes mellitus ] and glycemic control has been examined. Bouchta et al. studied patients who developed PTDM following renal transplantation and were converted from tacrolimus to cyclosporine, he found that Tacrolimus conversion to cyclosporin for PTDM is associated with a significant improvement in glucose metabolism and frequent reversal of diabetes [11].

In our study it was found that patients how were treated with Tacrolimus showed a significant increase in glucose level in compare to cyclosporine treated group. Figure (1), Table (1).

Regarding to the renal function tests elevations in serum creatinine and blood urea are common during cyclosporine therapy and do not necessarily indicate allograft rejection. A case of hemolytic uremic syndrome [HUS] associated with cyclosporine therapy has been reported. Another fatal case of acute tubular necrosis has been also reported. In addition, cyclosporine-induced hyperuricemia may predispose the patient to renal calculi [12].

In rare cases, long-term cyclosporine use has induced chronic kidney toxicity. Causing high blood urea or creatinine [13].

Serum creatinine and blood urea levels in cyclosporine treatment are not unusual to increase. These increases do not necessarily indicate rejection in renal transplants and before starting dosage adjustments, each patient should be fully evaluated [14].

In the study it was found that urea and creatinine concentration in both cyclosporine and tacrolimus treated group show un-significant differences in comparing to each other. Table (1)

Looking for the hematological changes that could be associated with renal transplant patient it was found that the incidence of leucopenia and neutropenia in kidney transplant recipients ranges from $10 \%$ to $55.5 \%$ and from $4.9 \%$ to $37.5 \%$, respectively [15].

The first year following transplant occurrence of neutropenia was 28 percent, and was significantly associated with tacrolimus combination therapy, according to a new retrospective study of kidney receivers. These results has been lined with this study in which WBC concentration in cyclosporin treated group found to be $12388.8 \pm 4168.16 / \mu \mathrm{L}$ while tacrolimus treated group shows a significant decrease to $7066.66 \pm 2003.33 / \mu L$. Figure (2)

Peter et al noted that initial reports had shown that tacrolimus (TC) therapy might have severe anemia. The degree of anemia under TC treatment in comparison to cyclosporine A (CsA) treatment after renal transplantation has been investigated. There is a correlation between $\mathrm{Hb}$ and creatinine clearance among children with functioning renal graft. There was no significant difference in the degree of anemia between children treated with TC- and CsA [16].

These results found to be compatible with our results that shown HB concentration for both cyclosporine and tacrolimus are lower than the normal range $8.2556 \pm 2.4 \mathrm{~g} / \mathrm{dL}$ and $6.9333 \pm 1.5 \mathrm{~g} / \mathrm{dL}$, respectively.

In conjunction with previous works, hyperuricaemia is more popular with treatment with cyclosporin, These findings consist of qualitative differences in cyclosporine and tacrolimus nephrotoxicity [17]. 
In isolated incidents, shortly after the medication was introduced in clinical usage, a salt loss nephropathy linked to Tacrolimus has been identified. Hyponatraemia has been reported in patient of combined pancreas with kidney transplants disease, and also was correlated to sodium losses in exocrine secretions because the pancreatic duct will drain into the bladder. A clinical study of kidney transplant recipients found that Tacrolimus was less associated with distal renal tubular acidosis than with Cyclosporin [18].

In our study it was found that calcium level was significantly lower than normal level with un-significant differences in potassium and sodium concentrations. Table (2)

\section{Conclusion}

Cyclosporine and tacrolimus and other immunosuppressant drugs which are used in kidney transplant patient can be selected depending on their efficiency and side effects associated with their use , tacrolimus treated patient has shown an increase in glucose level and decrease in WBC, hemoglobin and calcium level in comparing to cyclosporine treated group these result could help researcher and physician for better choice between immunosuppressant drugs depending on patient medical condition .

\section{ACKNOWLEDGEMENT: The researcher is thankful to the Biotechnology Research Center / Al -} Nahrain University for providing laboratory support and assistant to complete the requirements of this research.

\section{References}

1. Eckardt KU, Berns JS, Rocco MV, Kasiske BL. Definition and classification of CKD: the debate should be about patient prognosis--a position statement from KDOQI and KDIGO. Am J Kidney Dis. (2009); 53:915-20.

2. Levey AS, Andreoli SP, DuBose T, Provenzano R, Collins AJ. CKD: common, harmful, and treatable-World Kidney Day 2007. Am J Kidney Dis. (2007); 49:175-9.

3. Levey AS, Coresh J, Balk E. National Kidney Foundation practice guidelines for chronic kidney disease: evaluation, classification, and stratification. Ann Intern Med .(2003); 139:137-47.

4. EI Nahas M. The global challenge of chronic kidney disease. Kidney Int. (2005); 68 [6]:2918-2929.

5. Douglas C, John P. Vander's Renal Physiology . The McGraw-Hill Companies (2006); 3-7.

6. Myrray I, wilkison I, turmezei T. Oxford hand book of clinical medicine. New York: oxford university press. (2007); 294.

7. Wolfe RA, Ashby VB, Milford EL, Ojo AO, Ettenger RE, Agodoa LY, Held PJ, Port FK. Comparison of mortality in all patients on dialysis, patients on dialysis awaiting transplantation, and recipients of a first cadaveric transplant. N.Engl. J .Med.(1999); 341: 1725-1730.

8. Lippincott's illustrated reviews, Richard A.Harvey ,Immunosuppressants .

9. Naesens M, Kuypers DR, Sarwal M . Calcineurin inhibitor nephrotoxicity. Clin. J. Am. Soc. Nephrol.(2009); 4 [2]: 481-509.

10. Haddad EM, McAlister VC, Renouf E, Malthaner R, Kjaer MS, Gluud LL. Cyclosporin versus tacrolimus for liver transplanted patients. Cochrane Database Syst Rev. (2006);4:CD005161. 
11. Bouchta NB, Broeders N, Crenier L, Hoang AD, Abramowicz D, Wissing KM. Conversion from tacrolimus to cyclosporine A for new-onset diabetes after transplantation: a single-centre experience in renal transplanted patients and review of the literature. Transpl Int. (2008);21(2):146-51.

12. Jerry M, Oscar B, John F, Satoru T, Thomas E. starzl " treatment of cyclosporin-induced haemolytic uremic syndrome with fk506" Lancet. (1989); 2[8678-8679]: 1516.

13. Akinson K, Biggs JC, Hayes J, Ralston M, Dodds AJ, Naidoo D. Cyc1osporin A associated nephropathy in the first 100 days after allogeneic bone marrow transplantation: three distinct syndromes. $\mathrm{Br} \mathrm{J}$. Haematol. (1983);54:59-67.

14. Jordan ML, Shapiro R, Viyas CA, et al. FK506 "rescue" for resistant rejection of renal allografts under primary cyclosporine immunosuppression. Transplantation. (1994);57:860-865.

15. Zafrani L, Truffaut L, Kreis H, Etienne D, Rafat C. Incidence, risk factors and clinical consequences of neutropenia following kidney transplantation: A retrospective study. (2009); 9: 1816-1825.

16. Peter C1, Latta K, Graf D, Offner G, Brodehl J. Does tacrolimus cause more severe anemia than cyclosporine A in children after renal transplantation . Transpl Int.(1998) ;11:328-30.

17. Higgins R1, Ramaiyan K, Dasgupta T, Kanji H, Fletcher S, Lam F, Kashi H. Hyponatraemia and hyperkalaemia are more frequent in renal transplant recipients treated with tacrolimus than with cyclosporin. Further evidence for differences between cyclosporin and tacrolimus nephro-toxicities. Nephrol Dial Transplant. (2004);19[2]:444-50.

18. Heering P, Ivens K, Aker S, Grabensee B. Distal tubular acidosis induced by FK506. Clin Transplant MedlineWeb of Science.(1998); 12: 465-471. 